\title{
Effet de la température environnementale et du niveau d'activité métabolique sur la régulation à court terme de l'apport énergétique
}

Cécile Faure, Keyne Charlot, Stéphane Henri, Marie-Dominique Hardy-Dessources, Olivier Hue, Sophie Antoine-Jonville

- Introduction et but de l'étude. Alors que des millions de personnes sont exposées de façon chronique ou épisodique à des températures environnementales chaudes, les recommandations d'activité physique pour la santé métabolique et cardiovasculaire sont basées sur des études exclusivement réalisées en climat tempéré. L'objectif de ce projet est d'identifier les déterminants physiologiques de la régulation de la prise alimentaire à court terme chez l'adulte sain jeune et actif, dans différentes conditions de sollicitation métabolique (repos et exercice physique sous-maximal) et de température environnementale (neutre et chaude). Il s'agira ainsi d'étudier les signaux orexigènes et anorexigènes dans ces différentes conditions.

- Matériel et méthodes. Les participants volontaires étaient 9 jeunes hommes sains âgés de 20,6 $\pm 1,4$ ans (moyennetécart-type), et de bonne condition physique. Leur indice de masse corporelle était de $21,1 \pm 1,5 \mathrm{~kg} / \mathrm{m}^{2}$. L'étude clinique a été enregistrée auprès des autorités compétentes et a bénéficié des autorisations nécessaires (CPP13-018a/2013-A01037-38). Après une session d'évaluation et de familiarisation, ils ont tous participé à 4 sessions présentées en ordre aléatoire et réalisées à jeûn. Les sessions ont été réalisées dans 2 ambiances thermiques différentes (chaude : $31^{\circ} \mathrm{C}$ et neutre : $22^{\circ} \mathrm{C}$ ). Elles comprenaient une période test de 40 minutes, réalisée à 2 niveaux métaboliques (repos et exercice de pédalage à $65 \%$ de la consommation maximale d'oxygène), avec des périodes d'observation préalable et successive au test. La dernière phase de chaque session incluait un repas ad libitum. Des questionnaires, des prélèvements sanguins veineux et des mesures ont été réalisées à différents moments. Certains des participants $(n=6)$ ont également réalisé une épreuve d'hyperglycémie provoquée par voie orale dans les 2 ambiances thermiques. Des ANOVAs pour mesures répétées ont été effectuées.

- Résultats et analyse statistique. Le niveau d'activité métabolique $(p<0,01)$ influence l'apport énergétique relatif, sans que l'ambiance thermique ne modifie cet apport (effet simple : $p=0,741$; interaction avec le niveau métabolique : $p=0,442$ ) : l'apport énergétique relatif à la dépense est moins importante suite à l'exercice (717 $\pm 277 \mathrm{kcal}$ en moyenne pour les 2 climats) que dans la condition de repos $(979 \pm 285 \mathrm{kcal})$. Les évolutions au cours des sessions des taux sanguins de glucose et d'insuline sont affectées par l'ambiance thermique (effet ambiance thermique $x$ effet temps : $p=0,025$ ). Le niveau métabolique (exercice $v s$. repos) influence ces variables, mais ne module pas l'effet du climat. Différents biomarqueurs dosés dans cette étude sont potentiellement impliqués dans l'effet anorexigène relatif de l'exercice : c'est le cas du cortisol, de l'irisine, du peptide pancréatique, et de la cholécystokinine sur lesquels un effet du niveau métabolique est observé, sans interaction avec l'ambiance thermique. Le climat chaud est caractérisé par des valeurs post-prandiales d'insulinémie $(244 \pm 152 \mathrm{pmol} / \mathrm{L}$ et $379 \pm 253 \mathrm{pmol} / \mathrm{L}$ en température neutre, et chaude, respectivement) et de glycémie $(5,3 \pm 0,6 \mathrm{mmol} / \mathrm{L}$ et $6,0 \pm 0,6 \mathrm{mmol} / \mathrm{L})$ élevées suite au repos et à l'exercice. Ces données sont confirmées par des cinétiques différentes de réponse à l'hyperglycémie provoquée. 
- Conclusion : La régulation de la balance énergétique à court terme (après un exercice ou du repos) semble quantitativement indépendante de la température environnementale. En revanche, des adaptations physiologiques importantes impliquant l'insuline semblent se produire, et la glycorégulation paraît perturbée par l'exposition au climat chaud.

Conflit d'intérêt : aucun.

Financement : ce projet a bénéficié de financements du FSE et de la Région Guadeloupe (No CR/12-116), du ministère des outremers (No 0123-C001-D971/2013), ainsi que du Contrat de Projets Etat-Région impliquant des fonds FEDER (PO №1/1.4/-31793). 